\title{
Artificial Drug Delivery System for Diabetes
}

S. Geetha*

Department of Biomedical Engineering, Bharath University, Chennai, Tamil Nadu, India; geethag24@gmail.com

\section{Abstract}

Replacement of the endocrine functions of pancreatic $\beta$ cell presents a special challenge in substitute medicine to treat the major chronic non-communicable disease called diabetes mellitus in developing societies. A completely noninvasive diagnostic and therapeutic technique for diabetic patient can be possible with the help of bio medical equipments, so this completely implantable long term closed loop Insulin delivery systems are of great interest for biomedical therapy. With appropriate control algorithm, we can imitate behavior of mal functioning organs with highly specific, reliable, stable, \& long-lasting glucose sensor paves the new way for long-term therapeutic system for diabetes.

Keywords: Artificial Beta Cell, Glucose Sensor, Insulin Pump, Sensing System

\section{Introduction}

The term Artificial $\beta$ Cell is used exclusively for systems, aimed at replacing the endocrine function of the $\beta$ cell. By using various electronic components, we can construct a device which will replace the $\beta$ cell of pancreas. The $\beta$ cells, which occupy the central portion of the islet in Pancreas (Endocrine Part), comprise about $80 \%$ of its mass. They secrete insulin, a fuel-storing (anabolic) hormone that promotes sequestration of carbohydrate, Protein, \& fat in storage depots in the liver, muscle and adipose tissue. Endogenous Insulin secretion is greatest immediately after eating \& is lowest in the interval between meals. Co-ordination of insulin secretion with fluctuating demands for energy production results from $\beta$ cell stimulation by metabolites, other hormones, and neural signals. The beta cells monitor circulating solutes (in humans, primarily blood glucose) and release insulin in proportion to needs, in normal individuals. The increase in blood glucose levels on account of insulin deficiency leads to a disease called diabetes mellitus, which is the most common endocrine disease in developing societies, affecting as many as $12-15 \%$ of people in India. WHO has predicted that the number of diabetic patient Increases from 84 million to 228 million by the year 2025 . Depression is also a common psychiatric disorder among diabetic patients. Prevalence of depression and anxiety was more common among female patients ${ }^{8}$. Diabetes is a chronic systemic disease resulting from a disruption of fuel metabolism either because the body does not produce enough insulin, or because the available insulin is not effective. In either case, the result is accumulation of glucose in the blood or hyperglycemia, and once the renal tubular threshold for glucose is exceeded, a spillover of glucose into the urine or 'glycosuria' occurs. Hyperglycemia is thought to be the main determinant of microvascular alternation that affects several organs (renal glomerulus, retina, myocardium).

\subsection{Insulin Administration Systems}

\subsubsection{Syringes and Pens}

Insulin traditionally has been administered subcutaneously by means of syringe and needle from a vial containing insulin at a concentration of 40 units per $\mathrm{ml}$.

\subsubsection{Reservoirs, Depots, and Sprays}

Attempts have been made to develop distensible, implantable reservoirs or bags made of silicone elastomers, fitted with a small delivery catheter, and refillable transcutaneously. Insulin depots, in the form of bio-erodible polymer structures in which insulin-amorphous of its crystalline form-is entrapped and slowly released as hydrolytic decay of the carrier polymer progressively liberates it, presenting 
some of the same problems as reservoirs do. Nasal sprays of specially formulated insulin may become a practical modality of insulin therapy, if means are found to control reliably the dose administered. Use of standard insulin regimens results in high prevalence and large intra individual variation in hypoglycemia, particularly at night. Independent risk factors for nocturnal hypoglycemia were younger age, greater daily insulin dose, increasing weight ${ }^{7}$.

\subsubsection{Insulin Pumps}

Externally carried potable pumps are based on the motorized syringe or miniature roller pump designs. In actual practice, this system did not find wide acceptance on the part of patients, because it was just too cumbersome and socially unacceptable to adopt. The first implantable insulin pump was evaluated clinically in 1980. Unlike heparin delivery or cancer chemotherapy, insulin administration requires programmable pumps with adjustable flow rates. Implantable pumps provide better comfort for the patient than portable pumps, since the former are relatively unobtrusive and involve no danger of infection at the skin catheter junction. Technological improvements over 30 years of continuous SC infusion 9 have facilitated the development of insulin pumps, ready to be used in closed-loop systems ${ }^{3}$. The insulin pumps are small, can be remotely controlled and have alarms for cannula blockage, low battery, empty syringe, internal electronic failure, etc. They have a resolution up to $0.05 \mathrm{U} / \mathrm{h}$, suitable for toddlers receiving small amounts of insulin. However, insulin pumps do not alarm against leakage or dislodgement of the infusion cannula, which can complicate closed-loop control $^{4}$. Characteristics to consider when selecting a pump include the complexity of the pump relative to the user's abilities, water resistance, whether the pump is attached to the patient directly to the skin or via through tubing, whether or not the pump can communicate with a glucose monitor and/or sensor, and available diabetes data ${ }^{5}$.

\subsubsection{Artificial $\beta$ Cell}

It is the long term implantable closed loop insulin delivery systems, utilizing very recent advances in biomedical engineering. The function performed by this artificial beta cell is similar to the natural beta cell in terms of biological mechanisms involved in sensing, controlling \& delivering the insulin etc. This miniature device consists of an implantable pump, which has a reservoir containing insulin and a control valve that determines the flow rate of insulin into the surrounding tissue from which it is picked up by the circulation. The valve, in turn, is controlled by an electronic control system that performs the control algorithm for the device. A glucose sensor determines the body's glucose level and serves as the input device for the control system. Elevated glucose levels cause the pump to dispense increased amounts of insulin, while reduced glucose levels have the opposite effect. It is now possible to regulate blood glucose extracorporally using insulin infusion controlled by a glucose monitoring system known as artificial pancrease?.

\section{Materials and Methods}

The implantable artificial $\beta$ cell along with its catheters is designed to be entirely implanted between skins and muscle in the lateral abdominal area. This artificial $\beta$ cell consists of a number systems, such as

\section{Sensing system \\ Controlling system \\ Insulin delivery system \\ Power source system \\ Radio telemetry system}

The Long-Term Sensor System project, aiming at a fully implantable artificial beta cell, assessed the feasibility of glucose control by the combined implantation of a pump for peritoneal insulin delivery and a central intravenous glucose sensor close to the right atrium, connected via a subcutaneous lead toachieve an artificial endocrine

\section{Block Diagram}

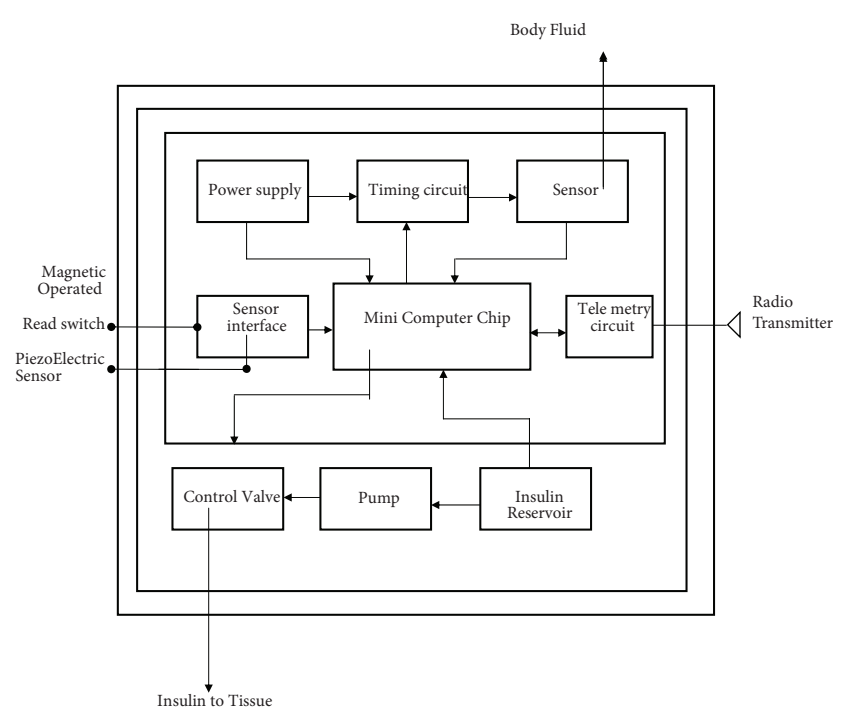

Artificial $\beta$ Cell 
pancreas, depends on the availability of three crucial components: 1) a safe and reliable device that delivers insulin continuously with a quick reactivity to change, 2) an accurate real-time continuous glucose monitoring system, 3) a control program to adapt insulin delivery according to blood glucose at all times ${ }^{1,2}$.

\section{Sensing System}

A glucose sensor provides a continuous reading of glucose concentration. The device consists of a detection part, which determines for the specificity of the glucose measurement, and a transducing element, which transforms the chemical or physical signals associated with glucose recognition into an electric signal.

\subsection{Electro Enzymatic Approach}

Glucose is recognized by a specific enzyme, usually glucose oxidase, layered on the anode of an electrode, generating hydrogen peroside, which is then oxidized and detected by the current generated in the presence of a fixed potential between the anode and the cathode. The major problem with this sensor is the instability of the immobilized enzyme and the fouling of the membrane surface under physiological conditions.

\subsection{Optical Approach}

A new fluorescence based affinity sensor. Near Infra red Absorption spectroscopic technique

\subsection{New Approach}

Sensor based on IV or oral administration /of contrast material specific to glucose.

Sensor based on implanting fluorescent nano spheres of polymer beads.

\subsection{Light Source}

We use Implantable LED as light source; the recently invented cheaper Zinc oxide lead can be used to provide desired wave length of light with low power consumption. Nano based new generation of LEDs has the capability of reducing the power used for lighting around the world by $90 \%$, because they are just highly efficient in converting electric power to light energy. Fiber-Optic catheter is used to detect changes in fluorescent light intensity, which is related to the concentration of glucose. This implantable fiber optic catheter must be a bio compatible one. The returning light with valuable information regarding glucose concentration is also carried by the same fiber optic catheter and, in term, falls on a photo detector. This part converts the light into electric Signal. The concept of developing an external monitor such as a wrist watch, which could receive its analyte from the implanted catheter and /or sensor and trigger a warning when glucose concentration is abnormally high or low ${ }^{6}$.

\subsection{Controlling System}

A built in computer or related control device is necessary to provide precise control of valve, which delivers the insulin at desirable rate. This must be accommodated in miniaturized device. The concept of accommodating control chip in micro $\mathrm{m}^{3}$ sized device will also be possible.

\subsection{Insulin Delviery System}

This system consists of insulin reservoir, pump and control valve. The feedback control signal from the chip stimulates the control valve to deliver the Insulin. The insulin is stored in a reservoir, which is made up of polymer matrix of suitable nano materials. The insulin should be in a highly concentrated form. The amount of glucose to be sensed by a sensor directly determines the amount of insulin to be delivered to the tissues. The insulin delivered and drains at a constant rate in to peritoneal cavity assuming uptake by the capillary beds in the serosal membranes that line the GIT, insulin reaches first the portal vein and then the liver which enhances its effectiveness. Primary care providers are now asked to render care to diabetic patients that once was the province of speacialist. The four area reviewed are pump training and education,infusion set change issues, skin issue and rate adjustment ${ }^{10}$.

\subsection{Power Source System}

The life of this device is determined by the current consumption of the electronic circuit.

Lithium cells: the long life lithium iodine battery powered device represents a significant advance in this technology.

Nickel metal hydride batteries: It is made of nano crystalline nickel, and metal hydrides require for less frequent recharging \& last much longer.

Recently, Nano Technologists have found generation of electricity while passing fluid through carbon nano 
tubes, so this idea can also be applied here to generate electric city from blood flow with the help of carbon nano tubes in order to miniature the device size \& longevity.

\subsection{Radio Telemetry System}

All the information regarding sensing, controlling, insulin delivering etc. can be monitored outside body by using wireless radio telemetry system. With the help of this telemetry system we can also be able to control the device with a hand held electronic module, or programs connected to the pump.

\subsection{Packaging of Implantable $\beta$ Cell}

This artificial $\beta$ cell is based on hybrid circuit technology because of its ability to compress complex electronic circuitry to a very small size. The hybrid circuit \& battery are hermetically sealed separately, and then both of them are sealed with in stainless steal case, thus providing complete and enduring isolation from the corrosive body environment. The case is manufactured either from titanium or from $316 \mathrm{~L}$ stainless steal.

The materials, exposed to subcutaneous environment are medical-grade epoxy, medical grade silicon rubber urethane, acetyl copolymer and type 316L stainless steel. All these materials have a long history in medical implants and have been discovered to be tissue compatible.

The hard wear resistant, corrosion resistant (biological fluids are corrosive) biocompatible, inert nano crystalline Zirconia ceramic and silicon carbide can be used as a best materials for this purpose ${ }^{5}$.

\section{Conclusion}

This idea of implanting miniaturised and encapsulated micro/nano electronic equipment paves a new era in substitute medicine. Thus, everything is possible under controllable and desirable condition with in certain limits.

An artificial beta cell regulates blood glucose concentration in a subject by continuously analyzing blood from the patient and deriving a computer output signal to drive a pump, which infuses insulin at a rate corresponding to the signal. A value of blood glucose concentration from the analyzed blood is used by a computer to determine a rate of change of this concentration, which in turn is used to derive a projected blood glucose level restoration of long-term normal blood glucose control in diabetic patients, supports the elaboration of an artificial beta cell. The possibility of implantation of the three crucial components of such a system (insulin delivery device, glucose sensor and controller) is analyzed. When insulin pump technology is combined with a continuous blood glucose monitoring system, the technology seems promising for real-time control of the blood sugar level. Currently, there are no mature algorithms to automatically control the insulin delivery, based on feedback of the blood glucose level. When the loop is closed, the system may function as an artificial pancreas.

\section{References}

1. Jaremko J, Rorstad O. Advances toward the implantable artificial pancreas for treatment of diabetes. Diabetes Care. 1998 Mar; 21(3):444-50.

2. Renard E. Implantable closed loop glucose-sensing and insulin delivery: the future for insulin pump therapy. Curr Opin Pharmacol. 2002 Dec; 2(6):708-16.

3. Sherr J, Tamborlane WV. Past, present, and future of insulin pump therapy: better shot at diabetes control. Mt Sinai J Med. 2008 Aug; 75(4):352-61.

4. Mirouze J, Selam JL, Pham TC, Cavadore D. Evaluation of exogenous insulin homeostasis by the artificial pancreas in insulin-dependent diabetes. Diabetologia. 1977; 13(3):273-78.

5. Pickup J, Keen H. Continuous subcutaneous insulin infusion at 25 years: evidence base for the expanding use of insulin pump therapy in type 1 diabetes. Diabetes Care. 2002; 25(3):593-98.

6. Wentholt IM, Hoekstra JB, Zwart A, DeVries JH. Pendra goes Dutch: lessons for the CE mark in Europe. Diabetologia. 2005; 48(6):1055-58.

7. Amin R, Ross K, Acerini CL, Edge JA, Warner J, Dunger DB. Hypoglycemia prevalence in prepubertal children with type 1 diabetes on standard insulin regimen: use of continuous glucose monitoring system. Diabetes Care. 2003; 26(3):662-67.

8. Shaban MC, Fosbury J, Kerr D, Cavan DA. The prevalence of depression and anxiety in adults with Type 1 diabetes. Diabetic Med. 2006 Dec; 23(12):1381-84.

9. Soeldner JS, Chang KW, Aisenberg S, Hiebert JM. Toward an implantable glucose sensor for the artificial beta cell. Temporal Aspects of Therapeutics. ALZA Conference Series. 1973; 2:181-207.

10. Lavin-Tompkins J. Insulin pump therapy: situations and solutions. Lippincotts Prim Care Pract. 1997 Nov-Dec; 1(5):519-26. 